\title{
SOME NOTES ON JENSEN-MERCER'S TYPE INEQUALITIES; EXTENSIONS AND REFINEMENTS WITH APPLICATIONS
}

\author{
LÁSZLÓ HORVÁTH
}

Abstract. In this paper we study inequalities corresponding to Jensen-Mercer's inequality. Some new extensions of Niezgoda's inequality and the integral version of Jensen-Mercer's inequality are given. The obtained inequalities do not only generalize the former ones, but our proofs are natural and simple. They clearly show the structure of such inequalities: they consist of two parts, a discrete or integral Jensen's inequality and then a majorization type inequality. Another purpose of the paper is to provide a deeper understanding of the methods used to refine Jensen-Mercer's and the corresponding inequalities. Moreover, some new refinements of these inequalities are obtained. Finally, some applications related to Fejér's and Hermite-Hadamard inequalities are given.

Mathematics subject classification (2020): 26D15, 26A51.

Keywords and phrases: Jensen-Mercer's inequality, discrete and integral Jensen's inequalities, refinements, Fejér and Hermite-Hadamard's inequalities.

\section{REFERENCES}

[1] M. Maqsood Ali, A. R. Khan, Generalized integral Mercer's inequality and integral means, J. Inequal. Spec. Funct. 10 (2019) No. 1 60-76.

[2] W. S. Cheung, A. Matković, J. PeČArić, A variant of Jessen's inequality and generalized means, J. Inequal. Pure Appl. Math. 7 (1) (2006) Article 10.

[3] S. S. DRAGOMIR, Improvements of Jensen-Mercer type discrete inequalities for convex functions on finite intervals, RGMIA Research Report Collection 23 (2020) Article 30, 12 pp.

[4] L. Fuchs, A new proof of an inequality of Hardy, Littlewood and Pólya, Mat. Tidsskr. B. 1947 (1947) $53-54$.

[5] L. HoRVÁTH, New refinements of the discrete Jensen's inequality generated by finite or infinite permutations, Aequat. Math. 94 (2020) no. 6, 1109-1121.

[6] L. HoRVÁtH, Refinements of the integral Jensen's inequality generated by finite or infinite permutations, J. Inequal. Appl. (2021) 2021:12, pp. 14

[7] J. Karamata, Sur une inégalité relative aux fonctions convexes, Publ. Math. Univ. Belgrade 1 (1932) $145-148$.

[8] M. Adil Khan, Asif R. Khan, J. PeČARIĆ, On the refinement of Jensen-Mercer's inequality, Rev. Anal. Numer. Theor. Approx. 41 (2012) 62-81.

[9] M. AdIL KHAN, J. PEČARIĆ, New refinements of the Jensen-Mercer inequality associated to positive n-tuples, Armen. J. Math. 12 (2020) no. 4, 1-12.

[10] Asif R. KhAn, J. PeČArić, M. PrAlJAK, A note on generalized Mercer's inequality, Bull. Malays. Math. Sci. Soc. 40 (2017) 881-889.

[11] P. LAH, M. Ribarič, Converse of Jensen's inequality for convex functions, Publ. Elektroteh. Fak. Univ. Beogr., Ser. Mat. Fiz. 412-460 (1973) 201-205.

[12] A. McD. Mercer, A variant of Jensen's inequality, J. Inequal. Pure \& Appl. Math. 4 (4) (2003) Article 73

[13] C. Niculescu, L. E. Persson, Convex functions and their applications, A contemporary approach, Springer, Berlin (2006) 
[14] M. NiezGodA, A generalization of Mercer's result on convex functions, Nonlinear Anal. 71 (2009) 2771-2779.

[15] Z. PAVIĆ, The Jensen-Mercer inequality with infinite convex combinations, Math. Sci. Appl. E-Notes 7 (2019) no. 1, 19-27.

[16] S. Simić, Some refinements of Hermite-Hadamard inequality, and an open problem, Kragujevac J. Math. 42 (2018) no. 3 349-356.

[17] Kuei-Lin Tseng, Shiow-Ru Hwang, S. S. Dragomir, Fejér-type inequalities (I), J. Inequal. Appl. Vol. 2010 Article ID 531976, 7 pp.

[18] Kuei-Lin Tseng, Shiow-Ru Hwang, S. S. Dragomir, New Hermite-Hadamard type inequalities for convex functions (II), Comput. Math. Appl. 62 (2011) 401-418.

[19] A. WitKOWS KI, A new proof of the monotonicity property of power means, J. Inequal. Pure \& Appl. Math. 5 (3) (2004) Article 73. 